\title{
THE
}

\section{Tracing molecular dephasing in biological tissue}

\author{
Mohammad Mokim \\ University of Rhode Island \\ Chris Carruba \\ University of Rhode Island \\ Feruz Ganikhanov \\ University of Rhode Island, fganikhanov@uri.edu
}

Follow this and additional works at: https://digitalcommons.uri.edu/phys_facpubs

The University of Rhode Island Faculty have made this article openly available.

Please let us know how Open Access to this research benefits you.

This is a pre-publication author manuscript of the final, published article.

Terms of Use

This article is made available under the terms and conditions applicable towards Open Access

Policy Articles, as set forth in our Terms of Use.

\section{Citation/Publisher Attribution}

Mokim, M., Carruba, C. \& Ganikhanov. (2017). Tracing molecular dephasing in biological tissue. Appl.

Phys. Lett. 111, 183701. doi: 10.1063/1.5001813

Available at: http://dx.doi.org/10.1063/1.5001813

This Article is brought to you for free and open access by the Physics at DigitalCommons@URI. It has been accepted for inclusion in Physics Faculty Publications by an authorized administrator of DigitalCommons@URI. For more information, please contact digitalcommons-group@uri.edu. 


\title{
Tracing molecular dephasing in biological tissue
}

\author{
M. Mokim ${ }^{1}$, C. Carruba ${ }^{1}$, and F. Ganikhanov ${ }^{1}$ \\ ${ }^{1}$ Department of Physics, University of Rhode Island, 2 Lippitt Road, Kingston, RI 02881, United
}

States of America.

\begin{abstract}
.
We demonstrate the quantitative spectroscopic characterization and imaging of biological tissue using coherent time-domain microscopy with femtosecond resolution. We identify tissue constituents and perform dephasing time $\left(T_{2}\right)$ measurements of characteristic Raman active vibrations. This was shown in subcutaneous mouse fat embedded within collagen rich areas of the dermis and the muscle connective tissue. The demonstrated equivalent spectral resolution $\left(<0.3 \mathrm{~cm}^{-1}\right)$ is an order of magnitude better compared to commonly used frequency-domain methods for characterization of biological media. This provides with the important dimension and parameter in biological media characterization and can become an effective tool in detecting minute changes in bio-molecular composition and environment that is critical for molecular level diagnosis.
\end{abstract}


Fundamental nonlinear optical phenomena have been shown to be useful in applications related to noninvasive characterization of biological media [1-4]. Raman scattering based techniques, both spontaneous and coherent versions, are of particular interest since their spectroscopic power can deliver molecular sensitive information that can become a key in early diagnosis of diseases. Absolute majority of the relevant applications of the techniques is, for natural reasons, in frequency domain. The coherent Raman microscopy studies were primarily applied to highlight tissue and cells constituent by producing high-contrast images at targeted Raman active vibration $[5,6]$. Spontaneous Raman version has been applied with greater focus towards detection of spectral features within cells and tissue [7]. However, the reported results have been limited to obtaining characteristic multi-line spectra and detecting relative changes in the intensities and spectral shifts with a goal to correlate those with biomolecular alterations occurring on sub-cellular level [8]. The true spectroscopic strength, that would ultimately include resolution of molecular vibration damping rates $\Gamma$ (or linewidths, $\Delta v=1 / \Gamma$ ) and line shapes, has not been enabled and demonstrated. It is worth noting that the damping rate is directly affected by inter- and intra-molecular interactions. Therefore, ability to measure Raman line shapes with a precision is absolutely crucial from that point of view. Depending on the immediate molecular environment (e.g. density, viscosity) and composition of tissue and cells, linewidths for the investigated Raman active modes can vary broadly. For instance, solute-solvent interactions in aqueous solutions can result in $0.3-7.5 \mathrm{~cm}^{-1}$ linewidth range that depend on the molar ratio [9]. Both spontaneous Raman and frequency-domain have the best possible resolution of $\sim 3-7 \mathrm{~cm}^{-1}$. The limits are imposed by the detection sensitivity in spontaneous Raman spectroscopy. Laser pulse bandwidths $\left(\sim 3-10 \mathrm{~cm}^{-1}\right)$ employed in coherent Raman microscopy does not provide a better resolution either. In addition, for the latter case the need to adjust laser wavelength in 
point-by-point spectral measurements adversely impacts the spectral resolution and precision. As a result, it becomes impossible to measure and detect Raman line shapes and bandwidths with a desired precision.

Alternative to the frequency-domain Raman scattering techniques is time-domain Coherent Anti-Stokes Raman Scattering (t-d CARS) spectroscopy. CARS signal can be viewed as a result of scattering of a probe pulse with amplitude $\mathrm{E}_{\mathrm{pr}}$ on the coherence built in the material. The initial coherence is the result of individual molecular displacements $\left(q_{i}\right)$ being phased in by the excitation pair of pulses $\left(E_{1}, E_{2}\right)$ with the difference frequency $\left(\omega_{1}-\omega_{2}\right)$ matching Raman active vibration $\left(\omega_{R}\right)$. The action results in macroscopic coherent amplitude $(Q)$. The coherence starts to decay freely in time as the consequence of various inter- and intra-molecular interactions that lead to changes in the phase (pure dephasing) and amplitude of the individual vibrations. Ensemble averaged solution for $Q$ contains autocorrelation function that yields in $\left\langle q(t) q\left(t^{\prime}\right)\right\rangle=\exp \left(-\left|t-t^{\prime}\right| / T_{2}\right)$ for the case of phase changing collisions that dominate the interactions. Constant $T_{2}$ represents characteristic dephasing time and the Raman active line is homogeneously broadened with the linewidth of

$$
\Delta v=1 / \pi c T_{2}
$$

expressed in wavenumber units.

CARS signal is detected at anti-Stokes frequency $\left(\omega_{a s}=\omega_{p r}+\omega_{1}-\omega_{2}\right)$. Non-resonant background can be more efficiently suppressed in t-d CARS down to the level of detector's dark which is different from the case of a coherent frequency-domain method. This gives an opportunity to detect really weak Raman active lines and potentially the ones that cannot be detected by spontaneous or coherent Raman spectroscopy. Tracing t-d CARS signal at longer delays (i.e. slower resonant part) within a decade translates to better than few percent precision 
in $T_{2}(\Delta v)$ measurements [10]. In other words, t-d CARS can result in the Green's function $(G(t))$ for the molecular system as a response to the ultrashort $\left(E_{1}(t), E_{2}(t)\right)$-pulse excitation. An ability to measure $(G(t))$ on extended time scale and within high dynamic range provides much more fine information about the corresponding Raman lineshape [10]. Lower limit for the equivalent spectral resolution is determined by one's ability to trace t-d CARS signal for as long time delays as possible.

The goal of this work is the direct dephasing time $\left(T_{2}\right)$ measurement of specific molecular vibrations within biological tissue. We demonstrate detection of time-domain replica within biological tissue, traced within more than one decade, for important and previously unresolved Raman signature line within fat cells. The ability to measure the corresponding dephasing times with high precision resulted in equivalent spectral resolution of better than $0.3 \mathrm{~cm}^{-1}$. This constitutes another important point since the achieved resolution is an order of magnitude better than the one that can be ultimately achieved by frequency-domain approaches applied to tissue or cell characterization. These highlight the strong potential of the time-domain approach with regard to biochemical and biomedical applications that seek reliable molecular level indicators for early disease diagnosis, etc. The only demonstration of the time-domain CARS microscopy showed lower sensitivity and was limited to artificial structures like polysterene beads probed at much stronger Raman resonance [11].

The ultrashort pulses $\left(E_{1}, E_{2}\right)$ are generated by independently tunable $(960-1120 \mathrm{~nm})$ optical parametric oscillators (OPO) at $76 \mathrm{MHz}$ repetition rate [12]. A small part of femtosecond Ti:sapphire laser output, that simultaneously pumped the OPOs, served as a third color pulse $\left(E_{p r}\right)$ that can be delayed. The three pulses are intrinsically synchronized in time.

For the case of biological tissue, generated SHG and CARS signals are detected in 
backward direction. SHG and CARS signals were filtered by the appropriate bandpass filter (BP) and diffraction grating (GR) with 1200 grooves $/ \mathrm{mm}$. The cooled PMT detector has a gain of up to $10^{7}$, high cathode sensitivity, and a dark current below $1 \mathrm{nA}$. The detected signal was digitized by data acquisition card. The card also provided synchronized analog signals to drive $x-y$ galvoscanners in order to generate raster scans for imaging. SHG and time-delayed CARS images can be generated with a spatial resolution of $300 \mathrm{~nm}$ using high-numerical aperture $(\mathrm{NA}=1.2)$ objective.

The tissue samples used in this investigation were dissected from above the longisimus dorsi muscle of C57BL/6 mice under after euthanasia with outer and inner surfaces of the adipose tissue identified. Slices of up to $100 \mu \mathrm{m}$ in thickness were fixed for $1 \mathrm{hr}$ in $4 \%$ PFA at $3^{\circ} \mathrm{C}$. The coverslips were treated with gelatin-chromium potassium sulfate solution for optimal tissue contact.

Fig.1 (a) shows a SHG image obtained with scanned fundamental beam, at optical frequency $\omega_{2}$, delivered by one of the OPOs tuned to $\sim 1095 \mathrm{~nm}$. The image shows a high SHG signal within the collagen type-II rich areas within the dermis and connective muscle tissue for which the second order optical nonlinearity is strong due to the lack of inversion center in the molecular structure of this type of protein.

There are fairly large areas in between the collagen areas where the SHG signal is absent. The collagen bundles could sustain $30-40 \mathrm{~mW}$ average power levels at this wavelength in the scanning mode of 2 frames/sec without the collagen fibrils being visibly altered or damaged. With the focused beam fixed on one spot within the collagen rich area a detectable damage could occur within the timeframe of few minutes. Figure 1(b) shows CARS image of the same area at zero time delay. The OPO wavelengths were tuned to $978 \mathrm{~nm}$ and $1095 \mathrm{~nm}$ respectively so that 
the targeted Raman active mode is at a frequency shift of $\omega_{1}-\omega_{2} \approx 1072 \mathrm{~cm}^{-1}$. Some collagen bundles seen in Fig. 1(a) can be still fairly well identified on the CARS image. However, the contrast is significantly lower with respect to surrounding areas. The image shows very strong signals coming from the areas where SHG signal was absent. These parts are filled with dense mouse fat as this was further confirmed by time-domain CARS measurements. Unlike the collagen, the structure of the fat molecules is centro-symmetric and therefore the areas with the fat are not seen on the SHG image. The CARS image clearly resolves a blood vessel with red blood cells (RBC) that does not have any trace in the SHG image. CARS and SHG images complement each other in a useful way. SHG imaging modality helped in positioning of the sample so that the other two beams can be blocked to minimize the risk of damage while we were identifying parts of tissue to be characterized. CARS image shown in Fig. 1(c) was obtained when the probe pulse was delayed by $200 \mathrm{fs}\left(\mathrm{t}_{\mathrm{d}}=200 \mathrm{fs}\right)$ with respect to the excitation pair. Both b) and c) images rather display frequency unspecific non-resonant CARS background. Indeed, the contrast ratio between fat tissue part and the remaining background remains about the same with a maximum value of about factor of 2.8 . When the delay is further increased beyond 500 fs the contrast drastically improves and the ratio of CARS signal within the fat tissue and the surrounding areas can exceed an order of magnitude (Fig. 1(d,e)).

Fig. 2(a) shows CARS signal versus delay time when Raman active vibrations within $\sim 1075 \mathrm{~cm}^{-1}$ vicinity were targeted. For this case the scanning area was reduced $\left(\sim 20 \times 20 \mu \mathrm{m}^{2}\right)$ to match large piece of fat located at around the center of the image shown in Fig 1(b). Signal fluctuations are fairly high and the signal-to-noise ratio is about factor of 5 despite the fact that the data were effectively averaged across more than 4000 pixel area. The data quality degraded further if the scanning was not performed and this has been the case for fairly moderate (i.e. $<25$ 
$\mathrm{mW}$ in combined power for the three beams) average power levels focused into the fat area. Some observations indicate that the tissue samples have been altered due to accumulated excess heat and high peak powers that lead to molecular ionization. The detailed study on this issue has not been performed. The obtained CARS transient clearly shows at least two spectral components that result in the coherent beat signal. The decay time is fairly long $(\sim 2 \mathrm{ps})$ and there is an indication of different decay times for the components. This can be noticed in decreased modulation depth for the beat signal at longer time delays. The obtained transients have been further analyzed by generating theoretical curves to fit the experimental data. We have applied a model that is based on time dynamics of the macroscopic coherent amplitude $(Q)$ [13]. Timedomain CARS signal can then be calculated by using the following formulae:

$$
\begin{aligned}
& S_{a s}\left(t_{d}\right)=\xi_{0} \int_{-\infty}^{\infty}|Q(t)|^{2} \varepsilon_{p r}^{2}\left(t-t_{d}\right) d t \\
& Q(t)=\int_{-\infty}^{\infty} G\left(t-t^{\prime}\right) \varepsilon_{1}\left(t^{\prime}\right) \varepsilon_{2}\left(t^{\prime}\right) d t
\end{aligned}
$$

In the equations above, $\varepsilon_{1,2, p r}(t)$ stand for unit area driving and probe pulses, $\xi_{0}$-detected antiStokes signal at zero delay, $G(t)$ - response (Green's) function of the corresponding vibrational system to $\delta$-pulsed driving fields. Applying certain solution algorithms for the above Fredholm type-I equations the $G(t)$ can be retrieved for arbitrarily shaped pulses [8]. We can also seek solution for $G(t)$ function for our case of i) Gaussian pulses and ii) when molecular collisions dominate the dephasing process. As was discussed in the introduction, the latter condition represents the case of homogenously broadened line. Therefore,

$$
G(t)=h(t) \sum_{j} A_{j} e^{-t\left(\frac{1}{T_{2 j}}-i \Delta \omega_{j}\right)}
$$


where $h(t)$ is Heaviside step function, $A_{j}$ - Raman line component amplitude, $T_{2 j}$-the component's dephasing time, $\Delta \omega_{j}$ - the component's shift from reference frequency (e.g. from $\left.\left(\omega_{1}-\omega_{2}\right)\right)$. By varying the above parameters we can find the best fit to our experimental data. For the vibrational modes in the vicinity of $\left(\omega_{1}-\omega_{2}\right) \approx 1072 \mathrm{~cm}^{-1}$ the best fit corresponded to the presence of two vibration lines with a frequency difference of $\Delta_{12}=\Delta \omega_{1}-\Delta \omega_{2}=28.7 \mathrm{~cm}^{-1}$, dephasing times of 2.6 and $1.7 \mathrm{ps}$ and the amplitude ratio of $A_{1} / A_{2}=19: 5$ respectively. The dephasing times $\left(T_{2 j}\right)$ obtained from our measurements suggest, if we apply formula (1), that the two homogenously broadened vibrations have linewidths of $\Delta v_{1}=4.1 \mathrm{~cm}^{-1}$ and $\Delta v_{2}=6.3 \mathrm{~cm}^{-1}$. Some comparison can be made with spontaneous Raman data available for fats $[14,15]$. We did not find any data on the relevant case that is obtained with a coherent frequency-domain technique. Spontaneous Raman spectroscopy of adipose tissue in mice has shown two not well resolved C-C bending vibrations within $\sim 1060-1120 \mathrm{~cm}^{-1}$ range sitting on a broad shoulder [15]. The line separation within the doublet at around $1080 \mathrm{~cm}^{-1}$ is not reported and could not be inferred from the data. The two, strongly overlapping and merging lines, show the combined width of about $25 \mathrm{~cm}^{-1}$ which is about the separation $\Delta_{12}$ that we found using our data. Thus, our results represent the only measurement of the linewidths and the spectral difference for the C-C vibrations in fat and clearly demonstrate the power of the time-domain method. Another feature that follows from our data is that the individual components with the doublet have different linewdth.

We next targeted higher-frequency and stronger line at $\sim 1265-1275 \mathrm{~cm}^{-1}$, assigned as a $=\mathrm{C}-\mathrm{H}$ bending vibration, to get a better comparison with available spontaneous Raman studies since the line is better resolved in them. Fig. 3 shows the transient signal when Raman active vibrations at around $\sim 1270 \mathrm{~cm}^{-1}$ frequency shift were excited and probed within the fat tissue. By 
fitting the data, using the model described above, we found that there are two closely spaced vibration modes at $1272 \mathrm{~cm}^{-1}$ with the frequency difference of $\Delta_{12}=48.5 \mathrm{~cm}^{-1}$, dephasing times of $550 \mathrm{fs}\left(\Delta v_{1}=19.3 \mathrm{~cm}^{-1}\right)$ and $670 \mathrm{fs}\left(\Delta v_{1}=15.8 \mathrm{~cm}^{-1}\right)$, and the amplitude ratio of 33:13. The study reported in [15] identifies this $\mathrm{C}-\mathrm{H}$ bending doublet positioned at $1264 \mathrm{~cm}^{-1}$ and $1301 \mathrm{~cm}^{-1}$ resulting in a frequency spacing of $35-40 \mathrm{~cm}^{-1}$ with linewidths that could not be obtained from the data. The doublet line frequency differences show some agreement with the one detected in our experiments. Overall, none of the Raman studies that we found on fatty acids, proteins, DNA [16] provided explicit line bandwidths. This is presumably due to the limited $\left(\sim 3-7 \mathrm{~cm}^{-1}\right)$ spectral resolutions and low scattering signals. Thus, we find our work to be the only report to reveal precise information on line separations and linewidths.

We have compared our results in mouse fat with identical time-domain CARS measurements that we performed in olive oil. Oils and lipids in fats have similar molecular composition and thus should have similar Raman active vibrational spectra. Raman active lines corresponding to C-C stretching vibration at $\sim 1070 \mathrm{~cm}^{-1}$ and scissoring $(\mathrm{C}-\mathrm{H})$ modes at $1267 \mathrm{~cm}^{-}$

${ }^{1}$ [17] were targeted. The corresponding results are shown in Fig. 4 (a) and (b). Since the data quality had been substantially better we were able to determine the parameters (see figure caption) more precisely. We point out again that a comprehensive comparison with data obtained by frequency- domain methods could not be performed since linewidths data were not available for the two modes in oil either. In general, from our experiments, fairly good agreement is found between fat and oil data as concerned linewidth and spectral separation parameters. However, the small differences, above spectral resolution, still noticeable. More detailed studies are needed, especially for different types of oils and fatty acids, in order to come up with a credible explanation for the detected differences. If we turn to the data for mouse fat again, one can see 
that there is a factor of 3-4 difference in linewidths for the targeted $\mathrm{C}-\mathrm{C}$ and $=\mathrm{C}-\mathrm{H}$ bending vibrations. We attribute this to the differences in character and lengths of the bonds and we think that those play a larger role in determining the dephasing times (damping rates, linewdith) for the vibrations compared to the heterogenous molecular environment.

In conclusion, we reported on implementation of spectroscopic imaging and characterization approach based on time-resolved version of CARS. We can identify tissue constituents and measure dephasing times for the associated vibrational modes. We show that the time-domain nonlinear microscopy of tissue delivers much more precise information on molecular fingerprints of the tissue constituents. Relating this type of information to diagnostics of diseases will be the task of major importance for future efforts.

\section{Acknowledgement}

Funding from NSF (DBI-1355307) is greatly appreciated. 


\section{References:}

${ }^{1}$ W. Denk, J.H. Strickler, and W.W. Webb, Science 105, 73 (1990).

${ }^{2}$ P.J. Campagnola, and L.M Leow, Nat. Biotechnol. 21, 1536 (2003).

${ }^{3}$ M. Zimmerley, P. Mahou, D. De'barre, M.Schanne-Klein, and E. Beaurepaire, Phys. Rev. X 3, $011002(2013)$.

${ }^{4}$ F.W. Christian, W. Min, B.G. Saar, S. Lu, G.R. Holtom, C. He, J.C. Tsai, J.X. Kang, and X.S. Xie, Science 322, 1857 (2008).

${ }^{5}$ C.L. Evans, E.O. Potma, M. Puoris'haag, D. Cote, C.P. Lin, and X.S. Xie, Proc. Natl. Acad. Sci. U.S.A 102, 16807 (2005).

${ }^{6}$ C.P. Pfeffer, B. R. Olsen, F. Ganikhanov , and François Légaré, J. Struct. Biol. 164, 140 (2008).

${ }^{7}$ W. Liu, H. Wangc, J. Du, C. Jing, Biosensors and Bioelectronics 97, 70 (2017)

${ }^{8}$ K. Eberhardt,C. Beleites, S. Marthandan, Ch. Matthäus, S. Diekmann, and J. Popp, Analytical Chemistry 89, 2937 (2017)

${ }^{9}$ K. Tanabe, J. Hiraishi, J. Raman. Spectrosc. 12, 274 (1982)

${ }^{10}$ A. Card , M. Mokim, and F. Ganikhanov, AIP Advances 6 , 025115 (2016)

${ }^{11}$ A.Volkmer, L.Book, and S.X. Xie, Appl. Phys. Lett. 80, 1505 (2002).

${ }^{12}$ J. D. Rowley, S.Yang, and F. Ganikhanov, J. Opt. Soc. Am. B 28, 1026 (2011).

${ }^{13}$ A. Laubereau, and W. Kaiser, Rev. Mod. Phys. 50, 607 (1978).

${ }^{15}$ G. Dobson, Eur. J. Lipid Sci. Technol. 103, 815 (2001)

${ }^{14}$ P. Meksiarun, B. B.Andriana, H. Matsuyoshi, and H. Sato, Sci. Rep. 6, 37068 (2016).

${ }^{16}$ G.J. Thomas, Annu. Rev. Biophys. Biomol. Struct. 28, 1 (1999)

${ }^{17}$ R. Korifi, Y. Le Dreau, J. Molinet, J. Artaud, and N. Dupuy, J. Raman Spectrosc. 42, 1540 (2011). 
Figure 1.
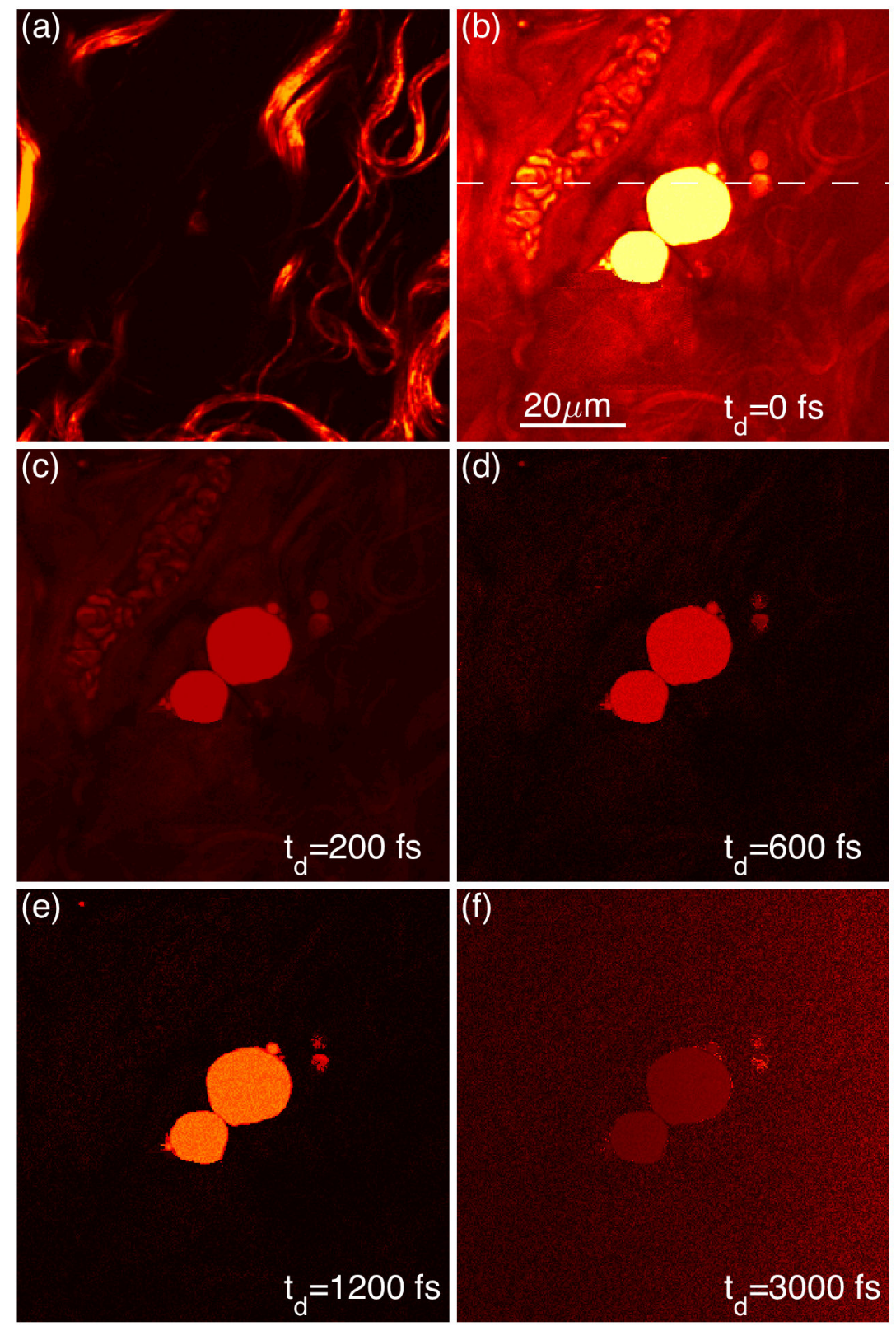

(a) SHG image obtained from the mouse dermis area; (b) CARS image of the same area at zero delay time between the probe $\left(E_{p r}\right)$ and driving $\left(E_{1}, E_{2}\right)$ pulses and $\omega_{1}-\omega_{2}=1072 \mathrm{~cm}^{-1}$. (c)-(f) CARS images at different delay times. 
Figure 2 .

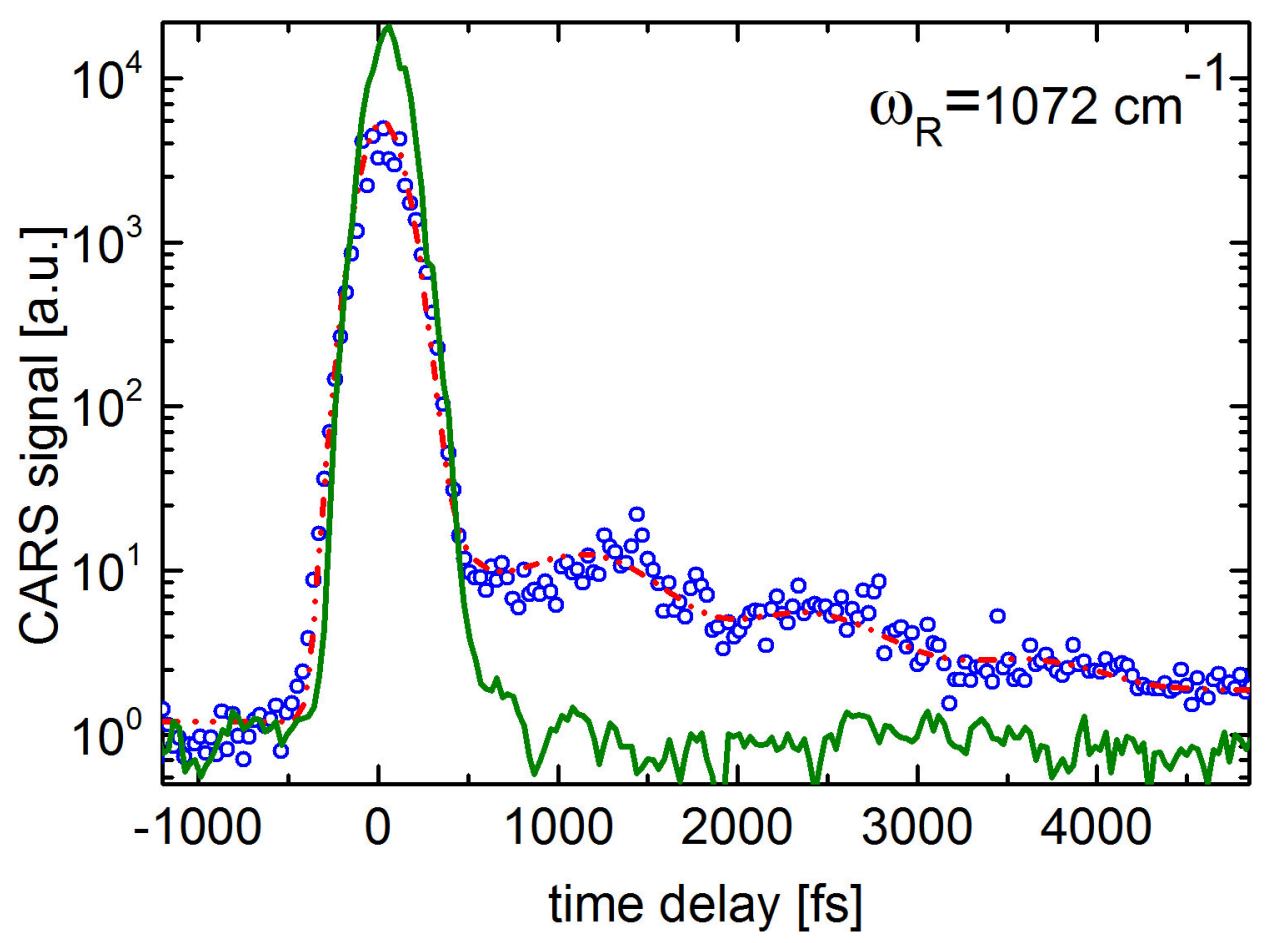

Time-domain CARS signal obtained from the $\sim 16 \mu \mathrm{m}$ diameter fat area located at the center of the mouse tissue for the image shown in Fig. 1(b). Solid green line represents non-resonant CARS signal obtained in microscope glass slide that was detected in the same (i.e. backward) direction and under the same other conditions. Dash dotted line represents the best fit to the data obtained by using formulae (2)-(4) and varying the corresponding line parameters (see text). 
Figure 3.

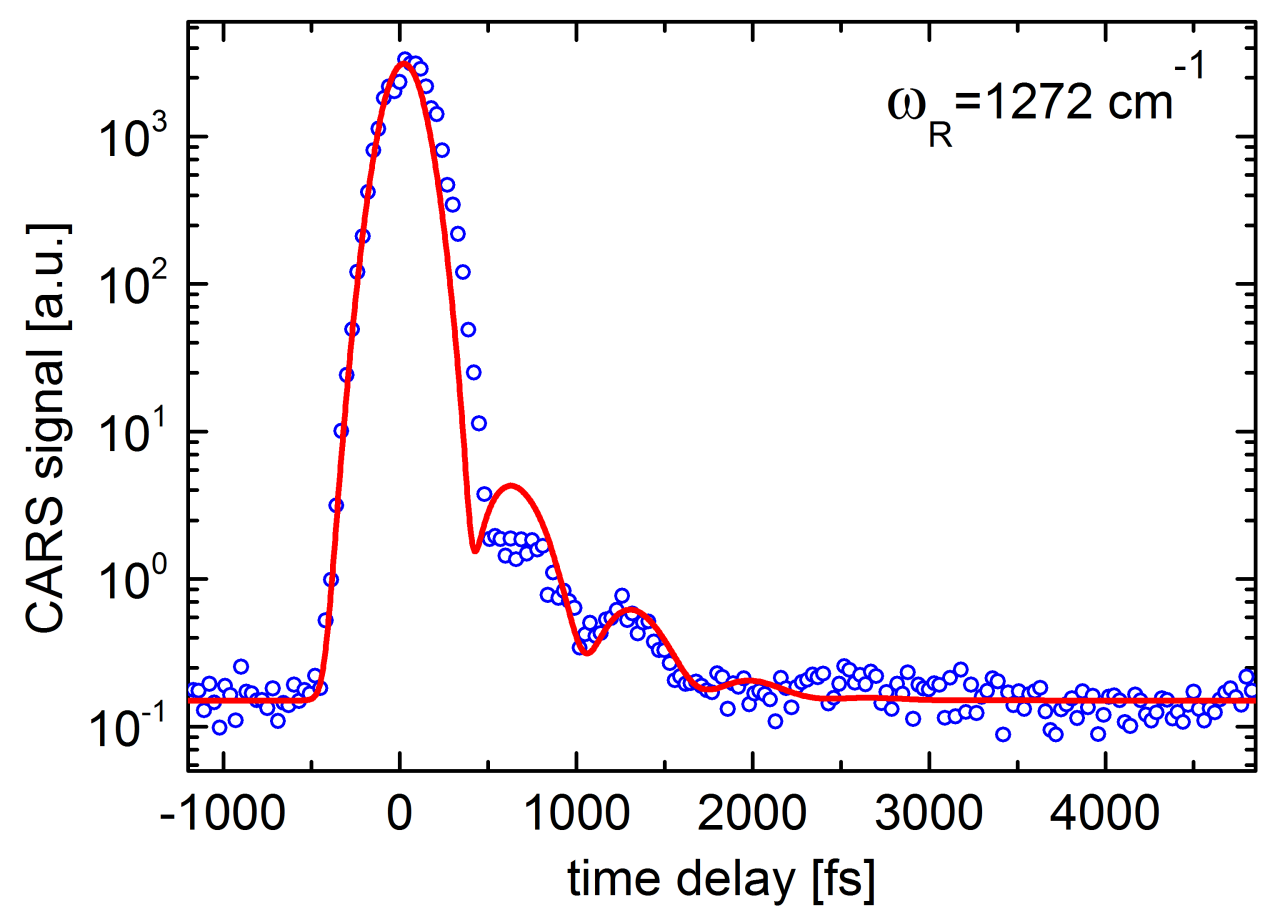

Time-domain CARS signal obtained from the mouse tissue area identified as a fat in the vicinity of Raman frequency shift of $\approx 1270 \mathrm{~cm}^{-1}$. Solid line represents the best fit to the data obtained by using formulae (2)-(4) and varying the corresponding line parameters (see text). 
Figure 4 (a), (b).
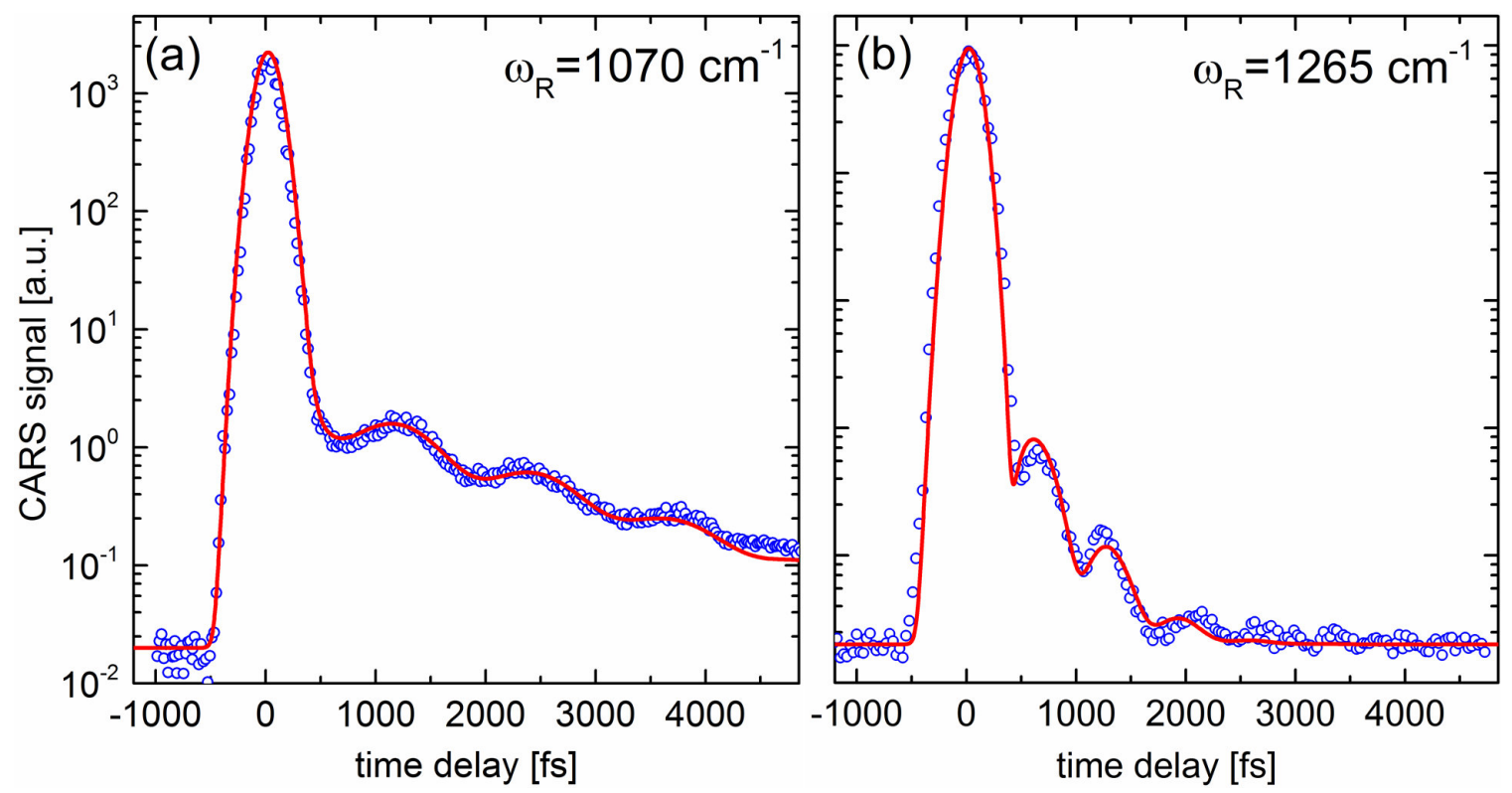

Time-domain CARS signals (open circles) obtained from a drop of olive oil. The Raman active vibrations near a) $\approx 1070 \mathrm{~cm}^{-1}$ and b) $1265 \mathrm{~cm}^{-1}$ were targeted and probed. The solid line represents the best fit to the data obtained using formulae (2)-(4) with the following parameters:

(a) $\Delta v_{1}=3.7 \mathrm{~cm}^{-1}, \Delta v_{2}=5.6 \mathrm{~cm}^{-1}, \Delta_{12}=23 \mathrm{~cm}^{-1}, \mathrm{~A}_{1} / \mathrm{A}_{2}=13 / 3 ;$ b) $\Delta v_{1}=21.6 \mathrm{~cm}^{-1}$, $\Delta v_{2}=17.5 \mathrm{~cm}^{-1}, \Delta_{12}=41.4 \mathrm{~cm}^{-1}, \mathrm{~A}_{1} / \mathrm{A}_{2}=11 / 3$. 